\title{
COVID-19 e complicações neurológicas: uma pequena revisão sistemática
}

\author{
COVID-19 and neurological complications: a small \\ systematic review
}

\section{COVID-19 y complicaciones neurológicas: una pequeña revisión sistemática}

\begin{abstract}
Arthur Santos Brandão ${ }^{1}$, Isabela de Nazaré Tavares Cardoso Souza ${ }^{2}$, Isadora Rocha Rosa ${ }^{3}$, Laíse Maria Barbosa Amaral ${ }^{4}$, Lucas Campos Maia5, Luigi Chermont Berni 6 , Maria Fernanda de Almeida Cavalcante Aranha7, Robson Leandro Sousa Andrade Júnior ${ }^{8}$, Thaynara Monteiro Paiva Garcia ${ }^{9}$, Rita de Cássia Silva de Oliveira ${ }^{10}$
\end{abstract}

\begin{abstract}
1.Discente de Medicina da Universidade do Estado do Pará (UEPA), Centro de Ciências Biológicas e da Saúde II (CCBS II), Belém-PA, Brasil. ORCID: https://orcid.org/0000-0002-8740-1987

2.Discente de Medicina da Universidade do Estado do Pará (UEPA), Centro de Ciências Biológicas e da Saúde II (CCBS II), Belém-PA, Brasil. ORCID: https://orcid.org/0000-0002-0158-404X

3.Discente de Medicina da Universidade do Estado do Pará (UEPA), Centro de Ciências Biológicas e da Saúde II (CCBS II), Belém-PA, Brasil. ORCID: https://orcid.org/0000-0001-6490-7881

4.Discente de Medicina da Universidade do Estado do Pará (UEPA), Centro de Ciências Biológicas e da Saúde II (CCBS II), Belém-PA, Brasil. ORCID: https://orcid.org/0000-0001-9024-8517

5.Discente de Medicina da Universidade do Estado do Pará (UEPA), Centro de Ciências Biológicas e da Saúde II (CCBS II), Belém-PA, Brasil. ORCID: https://orcid.org/0000-0003-0173-3436

6.Discente de Medicina da Universidade do Estado do Pará (UEPA), Centro de Ciências Biológicas e da Saúde II (CCBS II), Belém-PA, Brasil. ORCID: https://orcid.org/0000-0002-7762-2591

7.Discente de Medicina da Universidade do Estado do Pará (UEPA), Centro de Ciências Biológicas e da Saúde II (CCBS II), Belém-PA, Brasil. ORCID: https://orcid.org/0000-0002-5994-7122

8. Discente de Medicina da Universidade do Estado do Pará (UEPA), Centro de Ciências Biológicas e da Saúde II (CCBS II), Belém-PA, Brasil. ORCID: https://orcid.org/0000-0003-1893-762X

9.Discente de Medicina da Universidade do Estado do Pará (UEPA), Centro de Ciências Biológicas e da Saúde II (CCBS II), Belém-PA, Brasil. ORCID: https://orcid.org/0000-0003-3387-9106

10.Farmacêutica-Bioquímica, Doutora em Ciências Farmacêuticas (USP), Professora Adjunta do curso de Medicina, Departamento de Morfologia e Ciências Fisiológicas, Universidade do Estado do Pará (UEPA), Centro de Ciências Biológicas e da Saúde II (CCBS II), Belém-PA, Brasil. ORCID: https://orcid.org/0000$\underline{0003-3835-1183}$
\end{abstract}

\section{Resumo}

Introdução. A COVID-19 é uma doença provocada pela infeç̧ão de um beta-coronavírus conhecido como SARS-CoV-2, o qual desencadeia diversas manifestações relacionadas com vários sistemas orgânicos. Nesse contexto, complicações neurológicas foram descritas na literatura, as quais associaram vários distúrbios neurológicos com a infecção pelo SARS-CoV2. Objetivo. Deste modo, o objetivo do presente estudo consistiu em fazer uma pequena revisão sistemática, correlacionando a coVID-19 com neuropatologias neurodegenerativas. Método. Foram utilizadas as seguintes bases de dados: PUBMED, RIMA e LILACS. Os descritores selecionados foram encontrados no dicionário Descritores em Ciências da Saúde (DeCS) e Medical Subject Heading Terms (MeSH). Com isso, selecionou-se os seguintes: (COVID-19 OR SARS-CoV-2) AND (NEURODEGENERATION OR NEUROLOGIC COMPLICATIONS). Foram lidos os títulos e resumos de todos os préselecionados e retirados os que não se enquadravam no estilo metodológico. Excluiu-se dos achados: revisões sistemáticas com meta-análise, revisões sistemáticas com meta-síntese, e cartas. Vários motivos levaram a exclusão dos artigos, como: não causalidade entre o SARSCoV-2 e patologias neurológicas e a preexistência de condições neurodegenerativas nos indivíduos. Resultados. Os principais achados clínicos foram: Síndrome de Guillain-Barré, 
Acidente Vascular Cerebral, encefalopatias, hemorragias intracranianas, anosmia e algesia. Conclusão. Conclui-se, com a revisão, que os sintomas proporcionados pela COVID-19 podem variar e que nos casos mais graves, geram complicações neurodegenerativas. É cabível ressaltar que são necessárias mais investigações acerca da temática para ratificar a relação entre neurodegeneração e infecção por coronavírus.

Unitermos. COVID-19; SARS-CoV-2; Neurodegeneração; Complicações neurológicas

\begin{abstract}
Introduction. COVID-19 is a disease caused by beta-coronavirus known as SARS-CoV-2, which triggers several manifestations related to various organ systems. In this context, neurological complications have been described in the literature, which have associated several neurological disorders with SAR-CoV-2 infection. Objective. Thus, the aim of this study was to conduct a small systematic review, correlating COVID-19 with neurodegenerative neuropathologies. Method. The following databases were used: PUBMED, RIMA and LILACS. The selected descriptors were found in the Health Sciences Descriptors (DeCS) and Medical Subject Heading Terms (MeSH) dictionary. In addition, the descriptors following were selected: (COVID-19 OR SARS-CoV-2) AND (NEURODEGENERATION OR NEUROLOGIC COMPLICATIONS). The titles and abstracts of all those pre-selected were read and those that did not fit the methodological style were removed. The descriptors following were excluded from the findings: systematic reviews with meta-analysis, systematic reviews with metasynthesis and letters. Several reasons led to the exclusion of articles, such as: non-causality between SARS-CoV-2 and neurological pathologies and the pre-existence of neurodegenerative conditions in individuals. Results. The main clinical findings were: GuillanBarré syndrome, Stroke, encephalopathies, intracranial hemorrhages, anosmia and algesia. Conclusion. In conclusion, with this review, the symptoms provided by COVID-19 can vary and that in the most severe cases, they generate neurodegenerative complications. It is worth noting that further research on the subject is needed to confirm the relationship between neurodegeneration and coronavirus infection.
\end{abstract}

Keywords. COVID-19; SARS-CoV-2; Neurodegeneration; Neurologic complications

\title{
RESUMEN
}

Introducción. COVID-19 es una enfermedad causada por la infección de un beta-coronavirus conocido como SARS-CoV-2, que desencadena varias manifestaciones relacionadas con varios sistemas de órganos. En este contexto, se han descrito en la literatura complicaciones neurológicas que han asociado varios trastornos neurológicos con la infección por SAR-CoV-2. Objetivo. Por lo tanto, el objetivo de este estudio fue realizar una pequeña revisión sistemática, correlacionando COVID-19 con neuropatologías neurodegenerativas. Método. Se utilizaron las siguientes bases de datos: PUBMED, RIMA y LILACS. Los descriptores seleccionados se encontraron en el diccionario Health Sciences Descriptors (DeCS) y Medical Subject Heading Terms (MeSH). Con eso, se seleccionaron las siguientes: (COVID-19 O SARSCoV-2) Y (NEURODEGENERACIÓN O COMPLICACIONES NEUROLÓGICAS). Se leyeron los títulos y resúmenes de todos los preseleccionados y se eliminaron los que no se ajustaban al estilo metodológico. Se excluyeron de los hallazgos: revisiones sistemáticas con metanálisis, revisiones sistemáticas con meta-síntesis y cartas. Varias razones llevaron a la exclusión de artículos, como: la no causalidad entre el SARS-CoV-2 y las patologías neurológicas y la preexistencia de condiciones neurodegenerativas en los individuos. Resultados. Los principales hallazgos clínicos fueron: síndrome de Guillan-Barré, ictus, encefalopatías, hemorragias intracraneales, anosmia y algesia. Conclusión. Se concluye, con la revisión, que los síntomas que aporta el COVID-19 pueden variar y que en los casos más graves generan complicaciones neurodegenerativas. Vale la pena señalar que se necesitan más investigaciones sobre el tema para confirmar la relación entre la neurodegeneración y la infección por coronavirus.

Palabras clave: COVID-19; SARS-CoV-2; Neurodegeneración; Complicaciones neurológicas

Trabalho realizado na Universidade do Estado do Pará (UEPA), Belém-PA, Brasil.

Conflito de interesse: não Recebido em: 16/02/2021

Aceito em: 15/03/2021

Endereço para correspondência: Rita de Cássia S de Oliveira. Passagem Pinto Marques 164 casa 10. Bairro Castanheira. CEP 66645-550. Belém-PA, Brasil. E-mail: rita.oliveira@uepa.br 


\section{INTRODUÇÃO}

Em dezembro de 2019, surgiram os primeiros casos da COVID-19 na cidade de Wuhan, na China. Em fevereiro de 2020, nominou-se o agente da doença como coronavírus 2 da Síndrome Respiratória Aguda Grave (SARS-CoV-2). No mesmo ano, a Organização Mundial da Saúde (OMS) declarou estado de pandemia, provocando uma revolução na comunidade científica ${ }^{1}$.

A doença é ocasionada por um vírus de RNA de fita simples, envelopado e a união da proteína spike do vírus à Enzima Conversora de Angiotensina 2 (ECA2) desenvolve a infecção por SARS-CoV-2. Essa enzima possui expressividade, em especial, nos pulmões e no coração. Os sintomas respiratórios podem estar vinculados à secreção aumentada de ECA2 em pacientes com histórico de comorbidades. Ademais, o vírus afeta, predominantemente, células do epitélio alveolar².

Embora as percepções clínicas da COVID-19 sejam dominadas por sintomas respiratórios, manifestações neurológicas também foram comprovadas por estudos que detectaram: cefaleia, anosmia, ageusia, tontura e consciência prejudicada ${ }^{3}$. O Sistema Nervoso Central (SNC) possui receptores de ECA2, os quais foram encontrados em neurônios e células da glia. Tal fato é capaz de tornar compreensível como o SNC torna-se alvo do patógeno ${ }^{4}$.

Além disso, estudos apontam para a relação existente entre as manifestações neurológicas provenientes da infecção por SARS-CoV-2 e a neuroinflamação que progride 
com a tempestade de citocinas. Esse processo provém de uma desregulação da resposta imune inata, utilizada como primeira forma de defesa contra o SARS-CoV-2, por parte do organismo. Tal descontrole ocasiona um aumento dos níveis de citocinas e, consequentemente, pode provocar danos ao SNC, como alterações na neuroplasticidade e o desencadeamento de doenças neurodegenerativas no futuro ${ }^{4}$.

A partir dessas informações, o presente estudo tem por objetivo revisar o conteúdo bibliográfico disponível que exponha as manifestações neurológicas decorrentes da COVID-19. Com o intuito de agregar ao campo científico e fortalecer as discussões em torno dessa temática, busca-se mapear quais são as possíveis consequências neurológicas do SARS-CoV-2 nos indivíduos com testes positivos para COVID-19.

\section{MÉTODO}

\section{Caracterização da pesquisa e estratégias de busca}

O estudo consiste em uma revisão sistemática, a qual seguiu as recomendações Preferred Reporting Items for Systematic Reviews and Meta-Analyses (PRISMA). As buscas foram realizadas nas bases de dados PUBMED, Rede de Informática de Medicina Avançada (RIMA) e Literatura Latino-Americana e do Caribe em Ciências da Saúde (LILACS). 
Os descritores selecionados estão presentes nos dicionários Descritores em Ciências da Saúde (DeCS) e Medical Subject Heading Terms (MeSH), organizados nos seguintes operadores boleanos: (COVID-19 OR SARS-CoV2) AND (NEURODEGENERATION OR NEUROLOGIC COMPLICATIONS). Foi utilizado o filtro "Case Reports" ao realizar as buscas no PUBMED e LILACS.

\section{Critérios de seleção}

Critérios de inclusão

Os estudos selecionados são relatos de casos e séries de casos nas línguas inglesa, portuguesa e espanhola, indexados até os dias 05 de dezembro de 2020 nas bases de dados PUBMED e RIMA e até o dia 12 de dezembro de 2020 no LILACS.

Critérios de exclusão

Foram excluídas as revisões sistemáticas com meta-análise, com meta-síntese e cartas. Artigos que após a leitura do título e resumo não foram claros, que estavam indisponíveis, duplicatas, ou que não fossem em língua inglesa, portuguesa e espanhola também passaram por descarte.

\section{Análise de dados}

A partir das buscas, foram encontrados 180 estudos no PUBMED e realizou-se a leitura de 178 disponíveis em open acess. Foram incluídos 93 relatos de caso que abordassem 
complicações neurológicas reversíveis ou situações de neurodegeneração permanente que fossem efeito direto do COVID-19. Os demais artigos encontrados não foram adicionados por motivos diversos, como a não causalidade entre o SARS-CoV-2 e o patologia neurológica, a preexistência de condições neurodegenerativas nos indivíduos, não relatar danos neurológicos, não pertencer ao desenho metodológico estipulado, ser um relato pouco profundo, discorrer sobre a COVID-19 em animais e falta de confirmação do diagnóstico de COVID-19.

Não foi utilizado filtro "Relatos de caso" no RIMA, realizou-se somente a busca com os descritores, sendo encontrados 6 artigos, com 2 duplicados. Após a exclusão das duplicatas, restaram 4 artigos, foram lidos o título e o resumo, com a inclusão de apenas um, pois os demais não se tratava de relatos de casos.

Dada a escassez de estudos no LILACS, a busca foi realizada somente com os descritores (COVID-19 OR SARSCoV-2) e com o filtro de "Relato de caso", sendo encontrado 27 resultados. Entre os achados do LILACS, foram lidos os títulos e resumos, sendo incluído para leitura completa 3 que reportavam achados neurológicos decorrentes da COVID-19. Houve exclusão de 2 artigos porque os textos não estavam disponíveis. Entre os artigos lidos, um foi excluído por se tratar de uma galeria de imagens clínicas, o que não pertence ao desenho metodológico estipulado, e um por não relatar um quadro neurológico decorrente de COVID-19. 


\section{RESULTADOS E DISCUSSÃO}

A doença por coronavírus (COVID-19) detectada em dezembro de 2019 na China, se espalhou rapidamente pelo mundo implicando em uma declaração pela OMS de uma pandemia. Os sintomas típicos podem variar de doença respiratória leve a grave, somados a febre e tosse. Os grupos de risco para COVID-19 correspondem aos idosos e portadores de doenças crônicas. As complicações neurológicas em pacientes infectados com SARS-CoV-2 não foram amplamente relatadas, mas pacientes idosos possuem alto risco de desenvolver alterações no estado mental em infecções agudas, propiciando quadros de encefalopatias agudas e alterações no nível de consciência em casos de COVID-19. Estudos indicam que a incidência de acidente vascular cerebral agudo corresponde a 6\% dos casos, enquanto o comprometimento da consciência a $15 \%$ e a lesão do músculo esquelético a $19 \%^{5}$. Outras pesquisas apontam que o Líquido Cefalorraquidiano não apresenta componentes virais, designando que o vírus não é capaz de atravessar a barreira hematoencefálica, ou seja, apresenta a habilidade de bloquear meningite ou encefalite. Ademais, os pacientes testando positivo para a COVID-19 podem desenvolver encefalopatias durante a internação hospitalar de casos agudos ${ }^{6}$. Constatou-se, também, que a COVID-19 pode ter relação com encefalopatias raras, como a Encefalopatia Aguda Necrosante (ANE), que é caracterizada por múltiplas lesões simétricas no tálamo, corpo estriado, substância branca cerebral e tronco encefálico e ocorre de 
forma rápida e progressiva com convulsões, diminuição da consciência e vômitos, ocorrendo de 12 a 72 horas após o início dos sintomas da infecção viral ${ }^{7}$. Ainda há necessidade de uma maior investigação de uma potencial patologia no SNC relacionada a infecção por SARS-CoV-2, mas acreditase que uma resposta hiper inflamatória em pacientes com COVID-19 se relaciona com a síndrome do desconforto respiratório agudo (SDRA) que pode ser causada por uma exacerbada ativação macrofágica ou tempestade de citocinas $^{8}$. Além disso, casos de encefalopatias leves com lesão esplênio reversível em pacientes com anticorpos para SARS-CoV-2 também foram encontrados ${ }^{9}$.

Ainda no que tange aos resultados obtidos, também destacou-se o relato da hipótese da relação entre o receptor da ECA2 e manifestações neurológicas atreladas à COVID19, segundo a qual tal estrutura, presente no parênquima pulmonar, mucosa nasal, tratos renal, urinário e gastrointestinal, células gliais e neurônios, endotélio de vasos arteriais e venosos e nas células do músculo liso arterial, atua como porta de entrada para o vírus SARS-CoV2 e, devido às suas localizações, as complicações neurológicas seriam possíveis ${ }^{10}$. De maneira mais específica, o SARS-CoV-2 entra no sistema nervoso central por meio do suprimento sanguíneo, bulbo olfatório no teto da cavidade nasal ou de forma retrógrada, dos pulmões para o encéfalo, via nervo vago ${ }^{11}$, e infecta diversos tipos celulares por meio da passagem da proteína Spike do vírus para os receptores da ECA2, promovendo seus efeitos ${ }^{12}$. 
Além desse mecanismo indireto de efeito do SARS-CoV2 no sistema nervoso central, há a possibilidade de danos ocasionados por hipóxia, devido à falência respiratória, e através da atuação de interleucinas e citocinas. Somado a isso, o vírus pode ter também atuação direta, sendo essa via encefalite viral ${ }^{11}$.

Cabe destacar aqui um dos quadros clínicos com maior incidência foi a síndrome de Guillain-Barré (SGB) associada à COVID-19. Essa síndrome é uma doença desmielinizante de origem autoimune que ataca os nervos periféricos por meio da destruição da bainha de mielina, causando diminuição da velocidade dos impulsos nervosos e, em casos mais graves, ausência completa deles ${ }^{13}$. A síndrome é associada a algum estímulo imunológico, como infecções virais e bacterianas, e, com isso, sugere-se que o SARS-CoV2 possa desencadear uma resposta autoimune que levaria à destruição da bainha de mielina. Entretanto, esse mecanismo ainda não é claro, sendo necessários estudos que comprovem essa ação ${ }^{14}$.

A SGB também pode ser responsável por alguns quadros de insuficiência respiratória durante a infecção pelo novo coronavírus. Ao decorrer de um quadro de SGB é notada fraqueza muscular progressiva e em seguida paralisia muscular e, durante um caso grave, até os músculos respiratórios são afetados, levando à insuficiência respiratória ${ }^{13}$, sendo a incidência de falência respiratória de 20 a $30 \%$ entre os casos de SGB. Devido a essa taxa, alguns casos de COVID-19 podem ser atrelados à SGB e, com isso, 
a insuficiência respiratória pode estar ligada a fatores neuromusculares, o que leva a mudanças importantes na conduta clínica, tanto durante o tratamento da doença, quanto durante uma possível recuperação com fisioterapia ${ }^{14}$.

Outro quadro clínico frequente relacionado à COVID-19 é o de Acidente Vascular Cerebral (AVC), estando diretamente associado à tendência para trombofilia causada pelo vírus, com significativo aumento dos níveis de Dímero$\mathrm{d}^{15}$. De acordo com os estudos, os casos diagnosticados de COVID-19 seguidos de AVC são recorrentes até entre pacientes mais jovens e sem patologias prévias, de modo que é evidente a necessidade de estudos para compreender a associação entre $o$ vírus e as suas decorrentes complicações neurológicas ${ }^{16}$.

Ademais, a conduta clínica presente nos estudos envolve principalmente a administração de anticoagulantes e demonstra resultados favoráveis em diversas situações, com alguns pacientes totalmente curados e sem sequelas. Todavia, existem somente teorias acerca de um estado de hipercoagulação, sem comprovações, ressaltando a carência de pesquisas sobre os mecanismos envolvidos no desenvolvimento de AVC decorrente de COVID-1917.

A perda de olfato (anosmia) e a perda de paladar (ageusia), têm sido frequentemente relatadas como sintomas presentes em pacientes infectados com SARS-CoV2 sobretudo em paucissintomáticos ${ }^{18}$. A partir dos estudos de séries de casos, observou-se que tais sintomas se apresentam como os mais comuns relatados pelos pacientes, 
com um tempo mediano de apresentação de 3 dias após o início dos sintomas para anosmia e 2 dias para ageusia. Percebeu-se ainda que anosmia persistiu entre os pacientes por uma média de 11 dias (variando de 5 a 25 dias), enquanto ageusia perdurou por uma média de 8 dias (variando de 4 a 25 dias) ${ }^{19}$. Além disso, notou-se que esses sintomas são mais presentes em mulheres e jovens abaixo de 50,9 anos 20 .

Os receptores ECA2 podem estar diretamente relacionados com esses achados. Isso se deve ao fato de os receptores estarem mais presentes nos tecidos pulmonares, sobretudo em pneumócitos do tipo 2, e na mucosa oral da língua21. O SARS-CoV-2 utiliza esses receptores para a invasão celular, e inflamações nesses tecidos podem estar associados a anosmia e ageusia22,23. Ademais, disfunções olfativas e gustatórias são manifestações clínicas comumente presentes em diversas infecções virais, sobretudo aquelas que acometem o trato respiratório superior $^{24}$. Todavia, em pacientes com COVID-19, anosmia e ageusia não estão associadas diretamente à congestão nasal ou outros sintomas de rinite, logo esses sinais podem ser causados por danos diretos do vírus aos receptores olfativos e gustatórios ${ }^{25}$. Curiosamente, um dos estudos analisados na presente revisão sugere a hipótese de que um paciente portador de Espondilite Anquilosante (EA) acometido com a COVID-19 desenvolveu hiposmia e hipogeusia tardiamente, graças aos efeitos do medicamento Etanercepte, utilizado para o tratamento da EA. Porém, mais estudos são 
necessários para verificar se o agente afeta positivamente ou negativamente o curso da COVID-19 em humanos ${ }^{20}$.

O reconhecimento de paucissintomáticos é um fator importante para a quebra da cadeia de transmissão do vírus, o que torna fundamental a identificação de pacientes com anosmia e ageusia, haja vista que essa sintomatologia secundária e inespecífica pode ser a única manifestação do SARS-COV-2 nesses indivíduos ${ }^{25}$. Especialistas em rinologia sugerem que o aparecimento de tais sintomas deve atuar como sinalizador para o teste da doença, assim como um critério de auto isolamento ${ }^{26}$.

Além disso, outro fator observado com relativa frequência diante dos casos analisados é a hemorragia intracraniana. Ainda não são comprovados os modos como o SARS-CoV-2 atua para proporcionar tais hemorragias. Todavia, possíveis causas são pontuadas a partir da observação de diferentes casos de COVID-19 em que se notou manifestação desse quadro 27-30.

O SARS-CoV-2, ao se ligar a receptores da ECA2, reduz a atuação dessa enzima e gera uma série de consequências, como o aumento local da Angiotensina II, que reage com os receptores AT1 para aumentar a pressão sanguínea, a disfunção epitelial de vasos cerebrais, o que favorece hemorragias, e a redução da produção de Angiotensina 1-7, a qual desempenharia um papel neuroprotetor $e$ vasodilatador. Além da modificação epitelial, há o aumento da pressão sanguínea que, juntos, facilitam a ruptura de vasos e a ocorrência de hemorragias ${ }^{27}$, o que é somado com 
a privação de oxigênio nos tecidos como causa plausível desses episódios hemorrágicos, uma vez que alterações hidrostáticas e químicas na barreira hematoencefálica podem ocorrer, possibilitando o vazamento de eritrócitos ${ }^{28}$.

Além disso, as coagulações possivelmente causadas pelo SARS-CoV-2 em pacientes com COVID-19 deflagram a elevada taxa de Dímero-d, o qual é um produto resultado da reação de fibrinogênio em fibrina, demonstrando a ocorrência de coágulos no organismo como um todo. A partir disso, os fatores coagulantes do organismo são mais consumidos e, portanto, ficam menos disponíveis para em certas áreas do SNC, tornando os vasos sanguíneos desse sistema mais vulneráveis a sangramentos ${ }^{29}$.

A tempestade de citocinas é outro fator que supostamente é promovido pelo SARS-CoV-2, consistindo numa reação imunológica exagerada com elevada produção de citocinas pró-inflamatórias ${ }^{28,30}$. A partir disso, essa resposta em excesso passa a agredir o tecido dos vasos sanguíneos nervosos e, consequentemente, promover maior facilidade na ocorrência de hemorragias localizadas SNC ${ }^{30}$.

\section{CONCLUSÃO}

O SARS-CoV-2 acomete os indivíduos de várias faixas etárias com sintomas que podem variar em níveis de gravidade e sério comprometimento do sistema respiratório. Nos casos mais graves, complicações neurológicas podem se manifestar a partir da fisiopatologia que envolve a união da 
proteína Spike do vírus à ECA2 ou até mesmo uma ação viral direta no tecido nervoso. Os principais achados clínicos, que vão além da SDRA, foram Síndrome de Guillain-Barré, AVC, encefalopatias, hemorragias intracranianas, anosmia e algesia, destacados nesse levantamento bibliográfico sistemático apenas no ano de 2020. Dessa forma, a possibilidade de novas alterações neurológicas fisiopatológicas desencadeada pelo SARS-CoV-2 serão factíveis. Assim, há necessidade de mais estudos para melhor esclarecimento dos fatores que contribuam para o comprometimento neurológico dos pacientes com COVID19. Por fim, destaca-se a necessidade de avaliação precoce dos pacientes que testaram positivo para a COVID-19 e possuem tanto doença neurológica de base quanto aqueles que começaram a apresentar sinais e sintomas neurológicos, como cefaleia, confusão mental, parestesia e outros, sem doença ou quaisquer alterações neurológicas prévias, afim de evitar complicações futuras, buscando, dessa forma, a prevenção e o tratamento eficaz para um melhor desfecho clínico para os pacientes acometidos pela COVID-19.

\section{REFERÊNCIAS}

1.Marinho M, Souza S De, Araújo M, Farias DO, Rita A, Souza DO. Neurological complications of Covid-19: Narrative review. J Memor Med 2020;2:29-37. https://doi.org/10.37085/jmmv2.n1.2020.pp.29-37

2.Zheng YY, Ma YT, Zhang JY, Xie X. COVID-19 and the cardiovascular system. Nat Rev Cardiol 2020;17:259-60. http://doi.org/10.1038/s41569-020-0360-5

3. Needham EJ, Chou SHY, Coles AJ, Menon DK. Neurological Implications of COVID-19 Infections. Neurocrit Care 2020;32:667-71. https://doi.org/10.1007/s12028-020-00978-4 
4.Accorsi D, Chin CM, Santos I, Accorsi J, Bohac S. COVID-19 e o Sistema Nervoso Central. UL J Med 2020;1:81-7. http://revistas.unilago.edu.br/index.php/ulakes

5. Filatov A, Sharma P, Hindi F, Espinosa PS. Neurological Complications of Coronavirus Disease (COVID-19): Encephalopathy. Cureus 2020;12:e7352. https://doi.org/7310.7759/cureus.7352

6. Najjar S, Najjar A, Chong DJ, Pramanik BK, Kirsch C, Kuzniecky RI, et al. Central nervous system complications associated with SARS-CoV2 infection: integrative concepts of pathophysiology and case reports. J Neuroinflammation 2020;17:231. https://doi.org/10.1186/s12974020-01896-0

7. Mondolfi A, Bryce C, Grimes Z, Gordon R, Reidy J, Lednicky J, et al. Central Nervous System Involvement by Severe Acute Respiratory Syndrome Coronavirus-2 (SARS-CoV-2). J Med Virol 2020;92:699702. https://doi.org/10.1002/jmv.25915

8.Fara MG, Stein LK, Skliut M, Morgello S, Fifi JT, Dhamoon MS. Macrothrombosis and stroke in patients with mild Covid-19 infection. J Thromb Haemost 2020;18:2031-3. https://doi.org/10.1111/jth.14938 9.Barrios-López JM, Rego-García I, Muñoz Martínez C, Romero-Fábrega $J C$, Rivero Rodríguez $M$, Ruiz Giménez $J A$, et al. Ictus isquémico e infección por SARS-CoV-2, ¿asociación casual o causal? Neurología 2020;35:295-302. https://doi.org/10.1016/j.nrleng.2020.05.008

10. Hamming I, Timens W, Bulthuis MLC, Lely AT, Navis GJ, van Goor $\mathrm{H}$. Tissue distribution of ACE2 protein, the functional receptor for SARS coronavirus. A first step in understanding SARS pathogenesis. J Pathol 2004;203:631-7. https://doi.org/10.1002/path.1570

11.Li YC, Bai WZ, Hashikawa T. The neuroinvasive potential of SARSCoV2 may play a role in the respiratory failure of COVID-19 patients. J Med Virol 2020;92:552-5. https://doi.org/10.1002/jmv.25728

12. Hoffmann $M$, Kleine-Weber $H$, Schroeder $S$, Krüger $N$, Herrler $T$, Erichsen $\mathrm{S}$, et al. SARS-CoV-2 entry depends on ACE2 and TMPRSS2 and is blocked by a clinically proven protease inhibitor. Cell 2020;181:271-80. https://doi.org/10.1016/j.cell.2020.02.052

13. Machado ABM, Haertel LM. Neuroanatomia funcional. $3^{a}$ ed. São Paulo: Editora Atheneu. 2014.

14. Willison $\mathrm{HJ}$, Jacobs $\mathrm{BC}$, van Doorn PA. Guillain-Barré syndrome. Lancet 2016;388:717-27. https://doi.org/10.1016/S01406736(16)00339-1

15. Mao L, Jin $\mathrm{H}$, Wang $\mathrm{M}, \mathrm{Hu} \mathrm{Y}$, Chen $\mathrm{S}$, He $\mathrm{Q}$, et al. Neurologic Manifestations of Hospitalized Patients With Coronavirus Disease 2019 in Wuhan, China. JAMA Neurol 2020;77:683-90. https://doi.org/10.1001/jamaneurol.2020.1127

16.George J, Kirkland Z, Lattanzio N, Coleman J, Stone D. To Anticoagulate or Not: acute stroke in a covid-19 patient with gastrointestinal bleed. Cureus 2020;12:e10554. https://doi.org/10.7759/cureus.10554

17. Thachil J, Tang N, Gando S, Falanga A, Cattaneo M, Levi M, et al. ISTH interim guidance on recognition and management of 
coagulopathy in COVID-19. J Thromb Haemost 2020;18:1023-6. https://doi.org/10.1111/jth.14810

18. Vaira LA, Deiana G, Fois AG, Pirina P, Madeddu G, De Vito A, et al. Objective evaluation of anosmia and ageusia in COVID-19 patients: Single-center experience on 72 cases. Head Neck 2020;42:1252-8. https://doi.org/10.1002/hed.26204

19.Vargas-Gandica J, Winter D, Schnippe R, Rodriguez-Morales AG, Mondragon J, Escalera-Antezana JP, et al. Ageusia and anosmia, a common sign of COVID-19? A case series from four countries. J Neurovirol 2020;26:785-9. https://doi.org/10.1007/s13365-02000875-8

20.Lee J, Lee SJ. Olfactory and Gustatory Dysfunction in a COVID-19 Patient with Ankylosing Spondylitis Treated with Etanercept: Case Report. J Korean Med Sci 2020;35:e201. https://doi.org/10.3346/jkms.2020.35.e201

21.Xu $\mathrm{H}$, Zhong $\mathrm{L}$, Deng J, Peng J, Dan $\mathrm{H}$, Zeng $\mathrm{X}$, et al. High expression of ACE2 receptor of 2019-nCoV on the epithelial cells of oral mucosa. Inter J Oral Sci 2020;12:8. https://doi.org/10.1038/s41368020-0074-X

22. Lee $Y$, Min P, Lee S, Kim S. Prevalence and duration of acute loss of smell or taste in COVID-19 patients. J Korean Med Sci 2020;35:e174. https://doi.org/10.3346/jkms.2020.35.e174

23.Yan R, Zhang Y, Li Y, Xia L, Guo Y, Zhou Q. Structural basis for the recognition of SARS-CoV-2 by full-length human ACE2. Science 2020;367:1444-8. https://doi.org/10.1126/science.abb2762

24.Seiden AM. Postviral olfactory loss. Otolaryngol Clin North Am 2004;37:1159-66. https://doi.org/10.1016/j.otc.2004.06.007

25.Vaira LA, Salzano G, Deiana G, De Riu G. Anosmia and ageusia: common findings in COVID-19 patients. Laryngoscope 2020;130:1787. https://doi.org/10.1002/lary.28692

26.Russel B, Moss C, Rigg A, Hopkins C, Papa S, Hemelrijck M. Anosmia and ageusia are emerging as symptoms in patients with COVID-19: What does the current evidence say? Ecancer 2020;14:ed98. https://doi.org/10.3332/ecancer.2020.ed98

27.Pavlov V, Beylerli O, Gareev I, Torres Solis LF, Solís Herrera A, Aliev G. Covid-19-related intracerebral hemorrhage. Front Aging Neurosci 2020;12:352. https://doi.org/10.3389/fnagi.2020.600172

28. De Stefano P, Nencha U, De Stefano L, Mégevand P, Seeck M. Focal EEG changes indicating critical illness associated cerebral microbleeds in a Covid-19 patient. Clin Neurophysiol Pract 2020;5:125-9. https://doi.org/10.1016/j.cnp.2020.05.004

29.Becker RC. COVID-19 update: Covid-19-associated coagulopathy. J Thromb Thrombolysis

https://doi.org/10.1007/s11239-020-02134-3

2020;50:54-67.

30.Fajgenbaum DC, June $\mathrm{CH}$. Cytokine storm. Longo $\mathrm{DL}$, organizador. $\mathrm{N}$ Engl J Med 2020;383:2255-73.

https://doi.org/10.1056/NEJMra2026131 